\title{
Impact of oat processing on glycaemic and insulinaemic responses in healthy humans: a randomised clinical trial
}

\author{
Thomas M. S. Wolever ${ }^{1 *}$, Jodee Johnson ${ }^{2}$, Alexandra L. Jenkins ${ }^{1}$, Janice C. Campbell ${ }^{1}$, Adish Ezatagha ${ }^{1}$ \\ and YiFang $\mathrm{Chu}^{2}$ \\ ${ }^{1}$ Glycemic Index Laboratories Inc., Toronto, Ontario, M5C 2N8, Canada \\ ${ }^{2}$ Quaker Oats Center of Excellence, PepsiCo RED Nutrition, Barrington, IL 60010, USA
}

(Submitted 18 October 2018 - Final revision received 7 February 2019 - Accepted 8 February 2019 - First published online 9 May 2019)

\section{Abstract}

Oats can be processed in a variety of ways ranging from minimally processed such as steel-cut oats (SCO), to mildly processed such as largeflake oats (old fashioned oats, OFO), moderately processed such as instant oats (IO) or highly processed in ready-to-eat oat cereals such as Honey Nut Cheerios (HNC). Although processing is believed to increase glycaemic and insulinaemic responses, the effect of oat processing in these respects is unclear. Thus, we compared the glycaemic and insulinaemic responses elicited by $628 \mathrm{~kJ}$ portions of SCO, OFO, IO and HNC and a portion of Cream of Rice cereal (CR) containing the same amount of available-carbohydrate ( $23 \mathrm{~g}$ ) as the oatmeals. Healthy males ( $n$ 18) and females ( $n$ 12) completed this randomised, cross-over trial. Blood was taken fasting and at intervals for $3 \mathrm{~h}$ following test-meal consumption. Glucose and insulin peak-rises and incremental AUC (iAUC) were subjected to repeated-measures ANOVA using Tukey's test (two-sided $P<0 \cdot 05$ ) to compare individual means. Glucose peak-rise (primary endpoint, mean (SEM) mmol/l) after OFO, $2 \cdot 19$ (SEM 0.11), was significantly less than after CR, $2 \cdot 61$ (SEM 0.13); and glucose peak-rise after SCO, 1.93 (SEM 0.13), was significantly less than after CR, HNC, $2 \cdot 49$ (SEM 0.13) and IO 2.47 (SEM 0.13). Glucose iAUC was significantly lower after SCO than CR and HNC. Insulin peak rise was similar among the test meals, but insulin iAUC was significantly less after SCO than IO. Thus, the results show that oat processing affects glycaemic and insulinaemic responses with lower responses associated with less processing.

\section{Key words: Dietary carbohydrates: Dietary fibre: Oat $\beta$-glucan: Blood glucose: Insulin}

Oatmeal elicits a lower glycaemic response than most other types of ready-to-eat and cooked breakfast cereals when comparing equivalent amounts of available carbohydrate $(\operatorname{avCHO})^{(1-4)}$. Factors which may influence the glycaemic impact of cereals include the presence of soluble fibre and the effects of food processing. Oatmeal is rich in $\beta$-glucan, a highly viscous soluble dietary fibre found predominantly in the endospermic cell wall of oats and barley ${ }^{(5)}$ that has been shown to reduce glucose and insulin responses in normal ${ }^{(6-8)}$, hypercholesterolaemic ${ }^{(9)}$, hypertensive ${ }^{(10)}$ and diabetic ${ }^{(11-13)}$ subjects. Oat $\beta$-glucan is thought to reduce glycaemic responses by increasing the viscosity of the contents of the upper gut ${ }^{(6,7)}$, which, in turn, slows gastric emptying, reduces the rate of starch digestion and delays carbohydrate absorption by increasing the thickness of the so-called unstirred water layer in the small intestine $^{(14)}$.

Food processing includes treatments such as heat treatment, grinding and rolling. Moist heat gelatinises starch leading to more rapid digestibility and increased glycaemic response ${ }^{(15)}$.
Rolling crushes the grain which may disrupt cell walls and damage starch granules making them more available for digestion. Grinding disrupts the grain structure and reduces particle size, hence increasing the surface area to volume ratio; this, in turn, increases starch gelatinisation and the area available for attack by digestive enzymes ${ }^{(16)}$. However, in the case of oats, rolling and grinding may also make the $\beta$-glucan more bioavailable to reduce glycaemic responses.

A recent systematic review ${ }^{(1)}$ suggests that processing may affect the glycaemic impact of oats, with the mean glycaemic index (GI) of steel-cut oats, 53, and large-flake oats, 56, reported to be lower than those of quick-cooking oats, 71, and instant oatmeal (IO), 75. However, the source of the two GI values for steel-cut oats are websites from which details of the methods and results cannot be verified. In addition, the values for instant oats come mostly from the UK and Australia where the source of oats or the method of processing may differ from those used in North America. For example, we obtained a GI value of 67 (on the glucose scale) for instant oats purchased in

Abbreviations: avCHO, available carbohydrate; CR, Cream of Rice; GI, glycaemic index; HNC, Honey Nut Cheerios; iAUC, incremental AUC; inc2h, increment at $2 \mathrm{~h}$; IO, instant oatmeal; OFO, old fashioned oatmeal; RGR, relative glucose response; RIR, relative insulin response; SCO, steel cut oatmeal.

* Corresponding author: Dr T. M. S. Wolever, email thomas.wolever@utoronto.ca 
Canada $^{(4)}$ which is lower than 5 of the 7 GI values for quick cooking oats (range 61-80) and lower than 7 of the 9 values for instant oats (range 65-88) reported in the literature ${ }^{(1)}$. Thus, the effect of oat processing on the glycaemic and insulinaemic impact of oat products available in North America is not clear and needs to be established.

Therefore, our objective was to compare the glucose and insulin responses elicited by five breakfast cereals: a moderately processed control hot cereal made from rice (Cream of Rice (CR)) and four oat cereals processed in a variety of ways: minimally processed (steel cut oats), mildly processed (old fashioned oats (OFO)) moderately processed (IO) and highly processed (a ready-to-eat breakfast cereal made from oats). We hypothesised that the least heavily processed cereal, steel-cut oats, would elicit lower glucose and insulin responses than the more processed oat cereals and the control cereal.

\section{Methods}

We determined the glycaemic and insulinaemic responses elicited by five different cereals in male and non-pregnant female subjects ( $n$ 30) recruited from the pool of volunteers who previously participated in studies at GI Labs (20 Victoria Street, 3rd floor, Toronto) and had given permission to be contacted to be recruited for future studies. To be included subjects had to be aged 18-75 years, BMI $20 \cdot 0-34.9 \mathrm{~kg} / \mathrm{m}^{2}$, without diabetes (fasting plasma glucose $<7.0 \mathrm{mmol} / \mathrm{l}$ ) and be in good health. Subjects who regularly took supplements and medications not considered to influence glycaemic responses were allowed to participate. The research was conducted according to the Declaration of Helsinki, the protocol was approved by the Western Institutional Review Board ${ }^{\circledR}$ and each subject gave informed consent before their participation. The trial was registered at www.clinicaltrials.gov as NCT02616159.

\section{Study design and procedures}

A total of thirty eligible volunteers participated in this openlabel, randomised, cross-over design study on five separate days over a period of 2-5 weeks during the period from November 2015 to January 2016. The interval between successive tests was no $<48 \mathrm{~h}$ and no more than 2 weeks. On each test day, participants came to GI Labs in the morning after a 10$12 \mathrm{~h}$ overnight fast (water was allowed during this fasting period). Participants were asked to maintain stable dietary and activity habits throughout their participation in the study and to refrain from drinking alcohol, and from unusual levels of food intake or physical activity for $24 \mathrm{~h}$ before each test. On each test occasion, after subjects were weighed, two fasting blood samples were obtained by finger-prick $5 \mathrm{~min}$ apart and, after the second sample, the subject started to consume a test meal. The order of the test meals for each subject was randomly determined by a computer generated schedule. Subjects were asked to consume the entire test meal within 10 min. Each test meal was served with a drink of one or two cups of coffee or tea or water with $30 \mathrm{ml} \mathrm{2 \%}$ milk and non-energy sweetener if desired; the drink chosen remained the same for all study visits. At the first bite a timer was started and additional blood samples were taken at 15, 30, 45, 60, 90, 120, 150 and 180 min after starting to eat. At each time, 2-3 drops of blood were placed into a fluorooxalate tube for glucose analysis, and at every time except $150 \mathrm{~min}$, an additional 6-8 drops of blood were taken into a separate vial $(0.3 \mathrm{ml}$ microvette containing clot activator; Sarsted Inc.) for insulin analysis. If a participant's hands were cool, hands were warmed with an electric heating pad for 3-5 min before each sample. During the $3 \mathrm{~h}$ of the test, participants remained seated quietly. After the last blood sample participants were offered a snack and allowed to leave.

\section{Test meals}

The test meals consisted of Quaker IO (Quaker Oats Company), Quaker OFO, Quaker steel cut oatmeal (SCO), Honey Nut Cheerios (HNC; General Mills) and CR cereal (B\&G Foods Inc.). The four hot cereals were served in portions containing an equivalent amount of avCHO (23 g), but HNC was served in a portion containing the same amount of energy as the oatmeals. We matched HNC to the oatmeals for energy because it was recently reported that a $628 \mathrm{~kJ}$ serving of IO with $282 \mathrm{~kJ}$ lactosefree, fat-free milk, reduced appetite ${ }^{(17)}$ compared with $628 \mathrm{~kJ}$ of HNC plus $282 \mathrm{~kJ}$ lactose-free, fat-free milk. To see if these effects were related to differences in glucose and insulin responses, the same test meals were used in the present study. The portion sizes and composition of the test meals are shown in Table 1. IO, OFO, SCO and CR were prepared according to package instructions using $240 \mathrm{~g}$ water and served with $218.5 \mathrm{~g}$ lactose-free, fat-free milk (Natrel, Agropur Cooperative) as a beverage. Milk (218.5 g) was added to the HNC and served with $240 \mathrm{~g}$ water as a beverage. Each test meal was served with an additional drink of one or two cups of coffee or tea or water with $30 \mathrm{ml} 2 \%$ milk and non-energy sweetener if desired; at the

Table 1. Nutrient content of test meals*

\begin{tabular}{|c|c|c|c|c|c|c|c|c|}
\hline \multirow[b]{2}{*}{ Test Meal } & \multirow[b]{2}{*}{ Weight (g) } & \multirow[b]{2}{*}{ Energy (kJ) } & \multirow[b]{2}{*}{ Protein (g) } & \multirow[b]{2}{*}{ Fat $(\mathrm{g})$} & \multicolumn{4}{|c|}{ Carbohydrate (g) } \\
\hline & & & & & Total & Fibre & $B G$ & Available \\
\hline Instant oats & $40 \cdot 0$ & 628 & 5 & 3 & 27 & 4 & 1.79 & $23 \cdot 0$ \\
\hline Old fashioned oats & $40 \cdot 0$ & 628 & 5 & 3 & 27 & 4 & 1.72 & $23 \cdot 0$ \\
\hline Steel-cut oats & $40 \cdot 0$ & 628 & 5 & 2.5 & 27 & 4 & 1.74 & $23 \cdot 0$ \\
\hline Honey Nut Cheerios & $38 \cdot 2$ & 628 & $2 \cdot 7$ & $2 \cdot 1$ & 30 & $2 \cdot 7$ & 1.05 & $27 \cdot 3$ \\
\hline Cream of Rice & $28 \cdot 8$ & 427 & 1.9 & 0 & 23 & 0 & 0 & 23.0 \\
\hline Lactose-free, fat-free milk & 218.5 & 282 & $10 \cdot 1$ & 0 & $7 \cdot 6$ & 0 & 0 & $7 \cdot 6$ \\
\hline
\end{tabular}

$B G, \beta$-glucan.

${ }^{*}$ Available carbohydrate is total carbohydrate minus dietary fibre (rounded to nearest $0.1 \mathrm{~g}$ ). 
first visit each subject selected the type and volume of drink desired and the same type and volume was consumed on subsequent visits. The subjects had the option of adding $1 \mathrm{~g}$ of a sucralose sweetener (Splenda ${ }^{\circledR}$; Heartland Consumer Products) and one-half teaspoon of cinnamon to the oatmeal. If the subject added the Splenda and cinnamon to the oatmeal, they were required to add the same amounts of both to the HNC and CR.

\section{Biochemical analysis}

After collection the tubes containing blood for glucose analysis were rotated to mix the blood with the anti-coagulant, placed in a refrigerator until the last blood sample had been collected and stored at $-20^{\circ} \mathrm{C}$ until analysis which was performed within 3d using an YSI model 2300 STAT analyser. Typical values range for analytical $\mathrm{CV}$ for glucose in our hands is $<2 \%$. The microvette tubes containing blood for insulin were left at room temperature to allow the blood to clot, centrifuged and the serum transferred to labeled polypropylene tubes and stored at $-20^{\circ} \mathrm{C}$ before analysis of insulin using the Human Insulin EIA Kit (Alpco Diagnostics, catalogue no. 80-INSHU-E10.1). The lower limit of detection for this assay is $3.0 \mu \mathrm{U} / \mathrm{ml}$. The typical $\mathrm{CV}$ for serum insulin in our hands is approximately $7 \%$.

\section{Endpoints}

The pre-specified primary endpoint of the study was peakrise of blood glucose. The pre-specified secondary endpoints were peak-rise of serum insulin, incremental AUC (iAUC) from $0-3 \mathrm{~h}$ for glucose and insulin, peak blood glucose and serum insulin concentrations, and blood glucose and serum insulin concentrations at each time point measured. Other endpoints were glucose increment at $2 \mathrm{~h}$ (inc $2 \mathrm{~h}$ ), glucose below baseline at $2 \mathrm{~h}$ and the iAUC for glucose and insulin from $0-2$ and $2-3 \mathrm{~h}$.

\section{Calculations}

Data were entered into a spreadsheet by two different individuals and the values compared with assure accurate transcription. The incremental areas under the blood glucose and serum insulin response curves (iAUC), ignoring area below fasting, were calculated from $0-2,2-3$ and $0-3$ h using the trapezoid rule ${ }^{(18,19)}$. Peak concentration was the maximum concentration of glucose or insulin measured during the $3 \mathrm{~h}$ test; peak rise was peak minus the fasting concentration. Fasting glucose was taken to be the mean of the concentrations in the two fasting samples.

\section{Statistical analysis}

The primary endpoint (peak-rise of blood glucose) and other endpoints with only one value per treatment were analysed using repeated-measures ANOVA (RMANOVA). After demonstration of significant heterogeneity, individual means were compared using Tukey's test to adjust for multiple comparisons. The criterion for statistical significance was a two-tailed $P<0 \cdot 05$. Blood glucose and insulin concentrations were subjected to RMANOVA analysing for the main effects of time and test-meal and the time $\times$ test-meal interaction. After demonstrating a significant time $\times$ test-meal interaction for both glucose $\left(F_{36,1044}=4.41, P<0.0001\right)$ and insulin $\left(F_{32,928}=1 \cdot 77\right.$, $P=0.0055)$ the glucose and insulin concentrations at each time point were analysed by RMANOVA and Tukey's test as described above.

\section{Power analysis}

Although the primary endpoint of the study was peak rise in blood glucose, insufficient information was available about the expected differences in peak rise among the test meals to perform a power calculation. Therefore, study power was calculated based on glucose iAUC. The expected difference in iAUC among the test foods was based on the percentage difference in their GI values $(53,56,67,72$ and 82 , respectively for

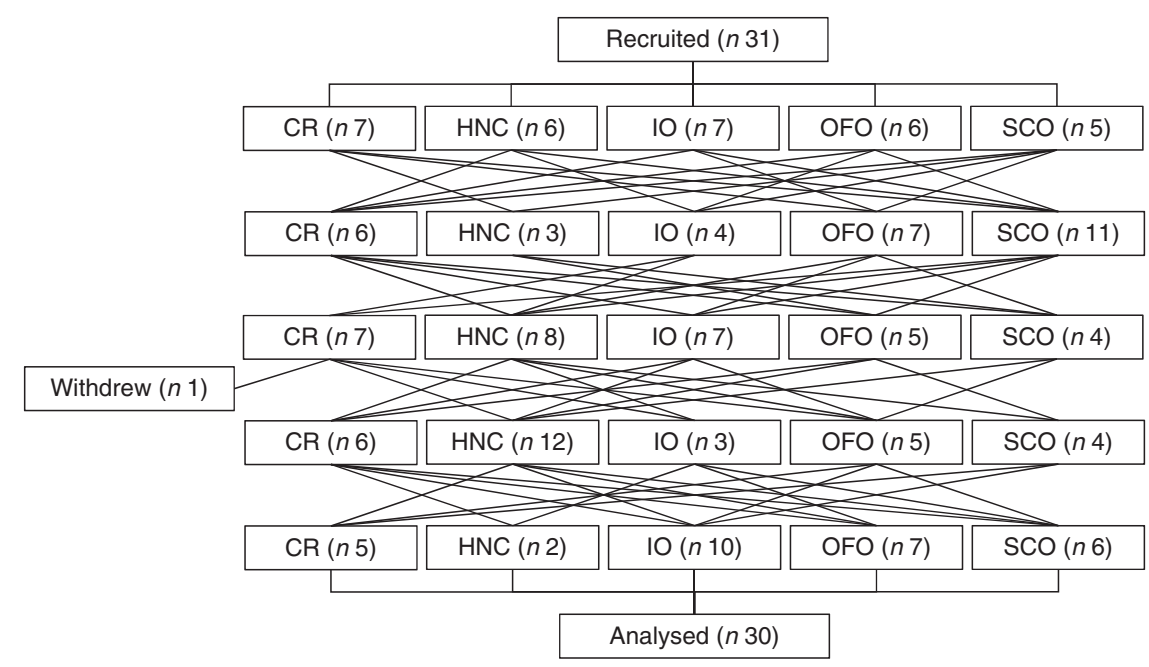

Fig. 1. Study flowchart. CR, Cream of Rice; HNC, Honey Nut Cheerios; IO, instant oatmeal; OFO, old fashioned oatmeal; SCO, steel cut oatmeal. 
SCO, OFO, IO, HNC and CR) taken from the international tables of $\mathrm{GI}^{(20)}$, our own GI value for $\mathrm{IO}^{(4)}$ and from the difference between oatmeal and CR from a previous study ${ }^{(21)}$. Study power was calculated using a within-subject SD of $23 \%$ and the $t$-distribution with two-sided $P<0 \cdot 05$. Based on this, $n 30$ subjects was chosen to provide $80 \%$ power to detect a $17 \%$ difference in iAUC (the estimated difference between OFO and IO), $90 \%$ power to detect a $20 \%$ difference in iAUC (the estimated difference between SCO and IO), and 95\% power to detect a $22 \%$ difference in iAUC (less than the estimated difference between SCO and NHC and CR).

\section{Results}

We recruited thirty-one subjects but one dropped-out for personal reasons after completing three tests leaving thirty subjects (eighteen males, twelve females) who completed the study (Fig. 1). These thirty subjects had mean age, 37 (SD 13) years; BMI, 24.9 (SD 3.0$) \mathrm{kg} / \mathrm{m}^{2}$; fasting blood glucose 4.44 (sD 0.44$) \mathrm{mmol} / \mathrm{l}$ and blood pressure 119 (SD 16)/71 (sD 11) $\mathrm{mmHg}$. A total of nineteen subjects took no prescription medications or supplements, four took supplements and seven were on the following daily medications: $20 \mathrm{mg}$ Adderall (amphetamine/dextroamphetamine) and an oral contraceptive ( $n$ 1); $100 \mathrm{mg}$ lamotrigine twice daily $(n 1) ; 300 \mathrm{mg}$ venlafaxine ( $n$ 1); an oral contraceptive ( $n$ 2); $0.5 \mathrm{mg}$ clonazepam $(n 1)$ and $20 \mathrm{mg}$ rivaroxaban ( $n$ 1). There were no adverse events reported during the study.

There was significant heterogeneity among treatments for glucose peak-rise (primary endpoint, $P<0.0001$ ) with the following significant differences among means: OFO less than $\mathrm{CR}$, and SCO less than CR, HNC and IO (Table 2). Peak glucose concentrations followed a similar pattern with SCO less than CR, HNC and IO. Blood glucose differed significantly among treatments at 30, 45, 60, 90 and $120 \mathrm{~min}$ (Fig. 2). There was significant heterogeneity among treatments for glucose iAUC from 0 to $2 \mathrm{~h}(P=0.0020)$ and iAUC from 0 to $3 \mathrm{~h}(P=0.010)$ with the following significant differences: iAUC 0-2 h, SCO less than CR, HNC and IO; iAUC 0-3 h, SCO less than CR and HNC (Table 2). Glucose iAUC from 2 to $3 \mathrm{~h}$ was similar among treatments. There was significant heterogeneity among treatments for glucose inc2h $(P=0.008)$ with inc2h being significantly higher after HNC and OFO than CR (Table 2). Glucose was significantly below fasting $2 \mathrm{~h}$ after $\mathrm{CR}$, but the difference from fasting was not significant for any of the other treatments. There was no significant difference in the number of subjects below baseline among the treatments by $\chi^{2}$ test (Table 2 ).

There was no significant difference in insulin peak-rise or peak concentration among treatments (Table 2), however, there were significant differences in serum insulin among treatments at 45 and $90 \mathrm{~min}$ (Fig. 2). There was significant heterogeneity among treatments in insulin iAUC $0-2 \mathrm{~h}(P=0.022)$ and iAUC $0-3 \mathrm{~h}$ $(P=0.022)$. Insulin iAUC after SCO was significantly less than after IO from $0-2$ and $0-3 \mathrm{~h}$ (Table 2 ). There was no significant difference in insulin iAUC from 2 to $3 \mathrm{~h}$ among treatments.

\section{Discussion}

The results show that SCO elicited a significantly lower glycaemic response (peak rise and iAUC $0-2 \mathrm{~h}$ ) than $\mathrm{CR}, \mathrm{HNC}$ and IO. Consistent with this, SCO also elicited a significantly lower insulinaemic response than IO. In addition, mean glucose peak rise after OFO was similar to that after SCO and significantly less than that after CR. However, mean glucose iAUC after OFO was not significantly less than that after CR because blood glucose after OFO remained elevated for longer, being significantly higher than CR at 90 and $120 \mathrm{~min}$. The similarity of the glycaemic responses elicited by IO and CR does not appear to be consistent with our recent finding that glucose iAUC after 30, 40 and $60 \mathrm{~g}$ portions of Quaker Quick Oats were each significantly less than those elicited by avCHO matched servings of CR by about $24 \%^{(21)}$. However, the difference in results may be because the variety of oats used in the previous study contained $38 \%$ more $\beta$-glucan than IO used here.

Table 2. Glucose and insulin peak-rises and incremental AUC

(Mean values with their standard errors, $n 30$ subjects)

\begin{tabular}{|c|c|c|c|c|c|c|c|c|c|c|}
\hline & \multicolumn{2}{|c|}{ Cream of Rice } & \multicolumn{2}{|c|}{ Honey Nut Cheerios } & \multicolumn{2}{|c|}{ Instant oatmeal } & \multicolumn{2}{|c|}{ Old fashioned oatmeal } & \multicolumn{2}{|c|}{ Steel cut oatmeal } \\
\hline & Mean & SEM & Mean & SEM & Mean & SEM & Mean & SEM & Mean & SEM \\
\hline \multicolumn{11}{|l|}{ Glucose } \\
\hline Peak-rise (mmol/l) & $2 \cdot 61^{\mathrm{a}}$ & $0 \cdot 13$ & $2 \cdot 49^{a, b}$ & 0.13 & $2 \cdot 47^{\mathrm{a}, \mathrm{b}}$ & 0.13 & $2 \cdot 19^{b, c}$ & 0.11 & $1.93^{\mathrm{C}}$ & 0.13 \\
\hline Peak concentration (mmol/l) & $7 \cdot 03^{a}$ & 0.13 & $6 \cdot 81^{\mathrm{a}}$ & 0.14 & $6 \cdot 84^{\mathrm{a}}$ & 0.15 & $6 \cdot 58^{a, b}$ & 0.11 & $6 \cdot 37^{\mathrm{b}}$ & 0.12 \\
\hline iAUC $0-2 \mathrm{~h}(\mathrm{mmol} \times \mathrm{min} / \mathrm{l})$ & $98^{\mathrm{a}}$ & 8 & $101^{\mathrm{a}}$ & 10 & $103^{\mathrm{a}}$ & 9 & $93^{a, b}$ & 10 & $68^{\mathrm{b}}$ & 6 \\
\hline iAUC $0-3 \mathrm{~h}(\mathrm{mmol} \times \mathrm{min} / \mathrm{l})$ & $100^{\mathrm{a}}$ & 8 & $109^{a}$ & 11 & $107^{\mathrm{a}, \mathrm{b}}$ & 9 & $99^{a, b}$ & 12 & $72^{\mathrm{b}}$ & 6 \\
\hline Glucose increment at $2 \mathrm{~h}(\mathrm{mmol} / \mathrm{l})$ & $-0.237^{b *}$ & 0.081 & $0.078^{a}$ & 0.090 & $-0.088^{a, b}$ & 0.064 & $0.100^{\mathrm{a}}$ & 0.080 & $-0.115^{a, b}$ & 0.062 \\
\hline Below fasting at $2 \mathrm{~h}$ & & & & & & & & & & \\
\hline$n$ & \multicolumn{2}{|c|}{20} & \multicolumn{2}{|c|}{14} & \multicolumn{2}{|l|}{17} & \multicolumn{2}{|c|}{12} & \multicolumn{2}{|c|}{18} \\
\hline$\%$ & \multicolumn{2}{|c|}{67} & \multicolumn{2}{|c|}{47} & 57 & & \multicolumn{2}{|c|}{40} & \multicolumn{2}{|c|}{60} \\
\hline \multicolumn{11}{|l|}{ Insulin } \\
\hline Peak-rise (pmol/l) & 312 & 39 & 328 & 38 & 353 & 41 & 303 & 40 & 290 & 38 \\
\hline Peak concentration (mmol/l) & 347 & 41 & 366 & 41 & 389 & 42 & 339 & 42 & 326 & 39 \\
\hline iAUC 0-2h (pmol × h/l) & $202^{a, b}$ & 31 & $204^{\mathrm{a}, \mathrm{b}}$ & 22 & $224^{\mathrm{a}}$ & 30 & $196^{\mathrm{a}, \mathrm{b}}$ & 32 & $168^{\mathrm{b}}$ & 23 \\
\hline IAUC 0-3h (pmol × h/l) & $205^{\mathrm{a}, \mathrm{b}}$ & 32 & $206^{a, b}$ & 22 & $228^{a}$ & 31 & $201^{a, b}$ & 32 & $172^{\mathrm{b}}$ & 23 \\
\hline
\end{tabular}

${ }^{a, b}$ Mean values with unlike superscript letters were significantly different $(P<0.05$, Tukey's test).

* Significantly different from fasting glucose (i.e. increment $=0$ ). 
(a)

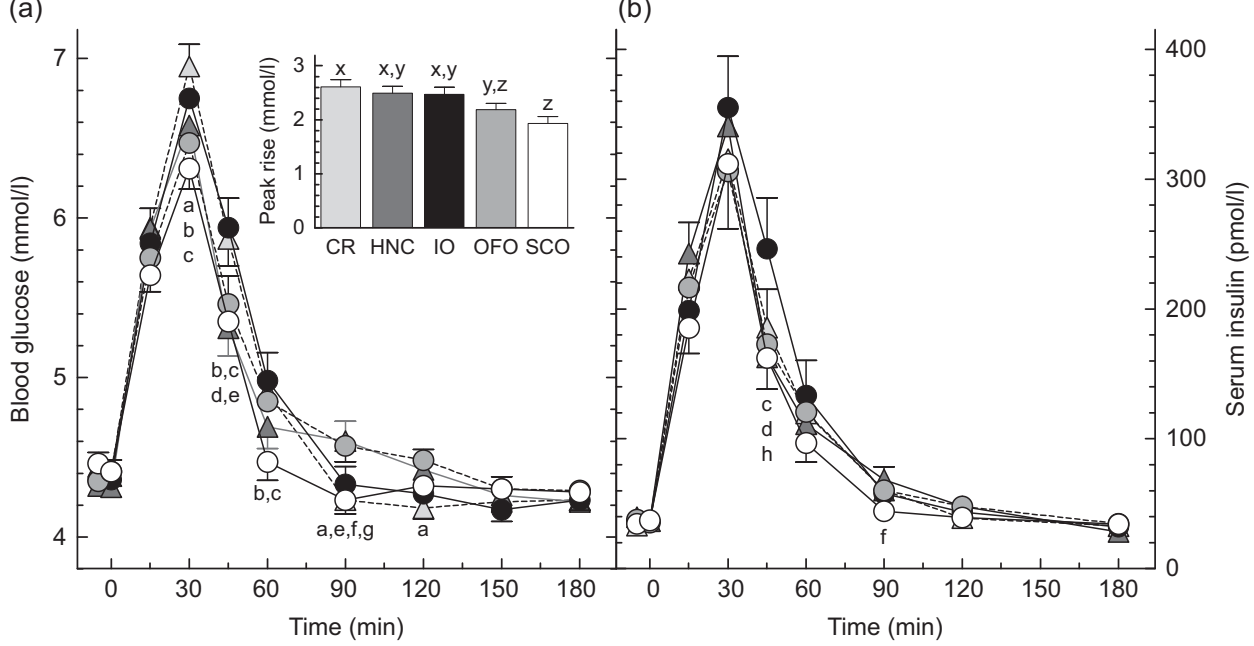

Fig. 2. Blood glucose (a) and serum insulin (b) responses elicited by the test-meals. Values are means for $n 30$ subjects, with their standard errors represented by vertical bars. Significant differences among means (Tukey's test, two-tailed $P<0.05)$ as follows: a, Cream of Rice (CR, --- $\triangle--)$ v. old fashioned oatmeal (OFO, ---O--); b, CR v. steel cut oatmeal (SCO, —- ); c, instant oatmeal (IO, —-) v. SCO; d, IO v. Honey Nut Cheerios (HNC, $\triangle$ ); e, CR v. HNC; f, HNC v. SCO; g, OFO v. SCO; h, IO v. OFO. Inset shows glucose peak rise. ${ }^{x, y, z}$ Bars with different letters differ significantly (Tukey's test, two-tailed $P<0.05$ ).

We expected that SCO, being the least processed oat cereal, would elicit the flattest glycaemic response; but this was not the case with OFO eliciting a flatter glycaemic response than SCO. Blood glucose after SCO fell quickly, being significantly less than all the other four cereals at either 60 or 90 min (Fig. 2) and significantly below fasting at $90 \mathrm{~min}$. This may indicate that some of the presumed avCHO in SCO was actually resistant starch. Due to the large particle size of SCO, some of its starch may have been trapped within intact cell walls or may not have been fully gelatinised during cooking and, thus, may not have been fully accessible for absorption from the small intestine. However, by $2 \mathrm{~h}$ glucose after SCO was not significantly different from baseline or from any of the other treatments.

Contrary to what might be expected from a cereal containing $45 \%$ of its avCHO as simple sugars, blood glucose after HNC remained elevated longer than $\mathrm{CR}$ and IO. The factors determining how long blood glucose remains elevated after eating are not well understood, but include the amount of avCHO consumed, the rate of avCHO absorption from the small intestine and the rate of uptake of glucose into tissues. We recently showed that, compared to a $30 \mathrm{~g}$ serving of oatmeal plus $9 \mathrm{~g}$ sucrose, consuming an avCHO matched portion ( $40 \mathrm{~g}$ ) of oatmeal without sugar elicited a flatter glycaemic response, with the same iAUC, but a lower peak rise and a prolonged elevation of blood glucose $\mathrm{e}^{(21)}$. If blood glucose inc $2 \mathrm{~h}$ is used as a measure of the time it takes glucose to reach baseline, inc $2 \mathrm{~h}$ after $\mathrm{HNC}$ and $\mathrm{OFO}$ were significantly greater than that after $\mathrm{CR}$ (Table 2). The longer elevation of blood glucose after HNC might have been due, at least in part, to the $14 \%$ greater amount of avCHO it contained compared to the other cereals (34.9v. $30.6 \mathrm{~g}$ ). Previous studies suggest that, as the amount of carbohydrate ingested increases, it takes blood glucose longer to return to baseline ${ }^{(22-25)}$. However, the relationship between time it takes blood glucose to return to baseline and the source and amount of avCHO consumed is not well understood and may depend on the nature of the subjects being tested. Previous studies $^{(23,24)}$ show that, across the range of $0-50 \mathrm{~g}$ avCHO intakes, inc $2 \mathrm{~h}$ tends to increase as avCHO intake from barley, bread and spaghetti increases, but decreases as avCHO intake from potato, fructose, sucrose and glucose increases (online Supplementary Fig. S1).

Nevertheless, the effect on increasing avCHO intake on the $0-2 \mathrm{~h}$ iAUC is well established with the $0-2 \mathrm{~h}$ iAUC elicited by a food, relative to that elicited by $50 \mathrm{~g}$ glucose (relative glucose response (RGR)) described by the following equation: RGR = $1.49 \times \mathrm{GI} \times\left(1-\mathrm{e}^{-0.0222 \mathrm{~g}}\right)$; where $\mathrm{g}$ is the grams avCHO consumed and GI is the food $\mathrm{GI}^{(26)}$. Based on this, the RGR for $30.6 \mathrm{~g}$ glucose $(\mathrm{GI}=100), 73.5$, is $91 \%$ of that for $34.9 \mathrm{~g}$ glucose, $80 \cdot 3$; thus, a portion of HNC containing the same amount of avCHO as the other cereals would be expected to elicit a $0-2 \mathrm{~h}$ iAUC $9 \%$ less than that of dose actually fed here, or $92 \mathrm{mmol} \times \mathrm{min} / \mathrm{l}$, a value which is similar to CR, IO and OFO, but perhaps no longer significantly greater than SCO.

Based on GI values in the literature ${ }^{(4,20,21)}$ which we used for the power analysis (HNC, 72; IO, 67; OFO, 56; SCO, 53 and CR, 82), we estimated that HNC, IO, OFO and SCO meals would elicit iAUC 0-2 h which were 88, 82, 68 and 65\%, respectively, of that elicited by CR. These can be compared to the observed relative responses of 90, 104, 92 and $72 \%$, respectively (online Supplementary Table S1; the value for HNC was reduced by $9 \%$ to adjust for the higher amount of avCHO it contained). The relative responses for SCO and $\mathrm{HNC}$ are similar to those predicted by GI, but those for IO and OFO are significantly higher than predicted. According to Tosh \& $\mathrm{Chu}^{(1)}$, the GI values for instant oats in the literature vary from 65 to 88 and that for rolled oats from 40 to 69 . Based on a between-lab SD of mean GI values of $9^{(27)}$ the probability that the minimum and maximum values of these ranges come from the same population is small ( 0.078 for instant oats and 0.030 for rolled oats). Thus, it is likely that the range of GI values for oats reported by Tosh and Chu represents real differences among the oats tested due to factors such as differences in processing (as demonstrated here) or 
differences in the amounts and physico-chemical properties of the $\beta$-glucan they contain due to differences in variety or environmental growing conditions ${ }^{(8,28,29)}$. If the GI of $\mathrm{CR}$ is taken to be 82 , the relative glycaemic response for IO could range from $100 \times 65 / 82=55 \%$ to $100 \times 88 / 82=106 \%$, a range which overlaps the $95 \% \mathrm{CI}$ of the relative glycaemic response for IO we observed $(94,115)$ (online Supplementary Table S1). Similarly, the range of expected relative glycaemic response for OFO, $100 \times 40 / 82=49 \%$ to $100 \times 69 / 82=84 \%$, overlaps the observed $95 \%$ CI $(79,106)$. This suggests that results we obtained are compatible with those in the literature.

The insulinaemic impact of foods is thought to be important to consider because, a reduced glucose response that is achieved at the expense of a high postprandial insulin response may not be beneficial due to the potentially deleterious health effects of hyperinsulinaemia ${ }^{(30)}$. Thus, both Health Canada and the European Food Safety Authority require that for a food to carry a claim related to a reduced glycaemic response, the insulinaemic response elicited by the food must not be disproportionately increased $^{(31,32)}$. Presumably, a disproportionate insulin response would be identified if the relative insulinaemic response elicited by a food, compared with control, was significantly greater than the relative glycaemic response compared with control. The mean relative insulin responses (RIR) were significantly higher than the respective RGR for HNC (121 v. $91 \%$ ) and SCO (92 v. 77\%) for peak rise (online Supplementary Table S1), but there were no differences between RIR and $R G R$ for $\mathrm{iAUC}_{0-2}$ and $\mathrm{iAUC}_{0-3}$. The ratios of RIR:RGR for $\mathrm{iAUC}_{0-2}$ found here varied between $1 \cdot 1$ and $1 \cdot 2$. However, the range of RIR:RGR ratios for $\mathrm{iAUC}_{0-2}$ for thirty-seven common non-dairy carbohydrate foods varies between about 0.7 and $1.4^{(33)}$ suggesting that variation of RIR:RGR within the range observed in this study is not physiologically significant.

Rebello et al. ${ }^{(17)}$ showed that, compared with $628 \mathrm{~kJ}$ of HNC plus $282 \mathrm{~kJ}$ milk, an equienergetic meal of IO plus milk significantly increased several subjective measures of appetite whereas an equienergetic meal of OFO plus milk did not; these results were associated with a significantly higher initial viscosity of IO compared with both HNC and OFO. Also, Rebello et al. $^{\left({ }^{34)}\right.}$ demonstrated that a meal of $1050 \mathrm{~kJ}$ from IO plus $471 \mathrm{~kJ}$ from milk elicited greater satiety as measured by less food intake at a subsequent meal than an equienergetic amount of HNC and milk. However, the present results showed that IO elicited a similar or, if anything, slightly higher glycaemic response than both $\mathrm{HNC}$ and $\mathrm{OFO}$, and a significantly higher serum insulin concentration than HNC and OFO at $45 \mathrm{~min}$. This is not consistent with the carbohydrates in IO being more slowly absorbed than those in HNC and OFO due to higher initial viscosity, and indeed is more in support of the concept that higher glucose and insulin responses promote satiety as quantified by reduced food intake ${ }^{(35)}$. Nevertheless, the glucose and insulin responses elicited by IO and HNC were similar and provide no obvious explanation for the differences in subjective appetite and food intake ratings found by Rebello et al. ${ }^{(17)}$. It should be noted in the 2016 study that objectively quantified reduced food intake ${ }^{(34)}$, Rebello et al. fed subjects $1050 \mathrm{~kJ}$ from the cereals and $471 \mathrm{~kJ}$ from milk; whereas the IO, OFO and HNC test meals we used here were the same as those used by
Rebello et al. in their 2014 study $^{(17)}$ (628 kJ from cereal and $282 \mathrm{~kJ}$ from lactose-free, fat-free milk).

We conclude that oat processing affects glycaemic and insulinaemic responses with lower responses associated with less processing. Similar glucose and insulin responses after $\mathrm{HNC}$, IO and OFO do not explain the differences in appetite and food intake previously described.

\section{Acknowledgements}

This research was funded by PepsiCo Inc. No in-kind support from PepsiCo was received by GI Labs or by any of its principals or employees. Pepsico contributed to the design of the study and provided comments on the manuscript, but had no role in the conduct of the study and no role in the analysis of the samples or data.

T. M. S. W. designed the study, wrote the protocol, conducted the statistical analysis, drafted the manuscript and takes overall responsibility for its content. T. M. S. W. considered the comments provided by the co-authors, but he determined the final content of the manuscript. Y. C. and J. J. conceived of the project, provided the test cereals, reviewed the manuscript, provided critical scientific input and approved the final version. A. L. J., J. C. C. and A. E. contributed to the design of the study, conducted the study and reviewed the manuscript, providing critical scientific input and approving the final version.

T. M. S. W. and his wife and A. L. J. are part owners of GI Labs Inc., a contract research organisation. T. M. S. W., his wife, A. L. J., J. C. C. and A. E. are employees of GI Labs; they have no rights to any intellectual property related to these results and have no financial interest in PepsiCo Inc., or any of its subsidiaries.

J. J. and Y. C. are employees of PepsiCo Inc., which manufactures oatmeal products under the brand name Quaker Oats ${ }^{\circledR}$. The views expressed in this article are those of the authors and do not necessarily reflect the opinion or policies of PepsiCo Inc.

\section{Supplementary material}

For supplementary material/s referred to in this article, please visit https://doi.org/10.1017/S0007114519000370

\section{References}

1. Tosh SM \& Chu YF (2015) Systematic review of the effect of processing of whole-grain oat cereals on glycaemic responses. Br J Nutr 114, 1256-262.

2. Jenkins DJA, Wolever TMS, Taylor RH, et al. (1981) Glycemic index of foods: a physiological basis for carbohydrate exchange. Am J Clin Nutr 34, 362-366.

3. Jenkins DJA, Wolever TMS, Jenkins AL, et al. (1983) The glycaemic index of foods tested in diabetic patients: a new basis for carbohydrate exchange favouring the use of legumes. Diabetologia 24, 257-264.

4. Wolever TMS, Katzman-Relle L, Jenkins AL, et al. (1994) Glycaemic index of 102 complex carbohydrate foods in patients with diabetes. Nutr Res 14, 651-669. 
5. Wood PJ (1986) Oat $\beta$-glucan: structure, location and properties. In Oats: Chemistry and Technology, pp. 121-152 FH Webstger, editor]. St Paul, MN: AACC Inc.

6. Wood PJ, Braaten JT, Scott FW, et al. (1994) Effect of dose and modification of viscous properties of oat gum on plasma glucose and insulin following an oral glucose load. Br J Nutr 72, 731-743.

7. Wood PJ, Beer MU \& Butler G (2000) Evaluation of role of concentration and molecular weight of oat $\beta$-glucan in determining effect of viscosity on plasma glucose and insulin following an oral glucose load. Br J Nutr 84, 19-23.

8. Regand A, Tosh SM, Wolever TM, et al. (2009) Physicochemical properties of beta-glucan in differently processed oat foods influence glycemic response. J Agric Food Chem 57, 8831-8838.

9. Biörklund M, van Rees A, Mensink RP, et al. (2005) Changes in serum lipids and postprandial glucose and insulin concentrations after consumption of beverages with $\beta$-glucans from oats or barley: a randomized dose-controlled trial. Eur $J$ Clin Nutr 59, 1272-1281.

10. Maki KC, Galant R, Samuel P, et al. (2007) Effects of consuming foods containing oat $\beta$-glucan on blood pressure, carbohydrate metabolism and biomarkers of oxidative stress in men and women with elevated blood pressure. Eur J Clin Nutr 61, 786-795.

11. Tapola N, Karvonen H, Sarkkinen E, et al. (2005) Glycemic responses of oat bran products in type 2 diabetic patients. Nutr Metab Cardiovasc Dis 15, 255-261.

12. Kabir M, Oppert JM, Vidal H, et al. (2002) Four-week low glycemic index breakfast with a modest amount of soluble fibers in type 2 diabetic men. Metabolism 51, 819-826.

13. Jenkins AL, Jenkins DJA, Zdravkovic U, et al. (2002) Depression of the glycemic index by high levels of beta-glucan fiber in two functional foods tested in type 2 diabetes. Eur J Clin Nutr 56, 622-628.

14. Jenkins DJA, Wolever TMS, Leeds AR, et al. (1978) Dietary fibres, fibre analogues, and glucose tolerance: importance of viscosity. Br Med J 1, 1392-1394.

15. Rosenthal SM \& Ziegler EE (1929) The effect of uncooked starches on the blood sugar or normal and of diabetic subjects. Arch Int Med 44, 344-350.

16. Jenkins DJA, Wesson V, Wolever TMS, et al. (1988) Wholemeal versus wholegrain breads: proportion of whole or cracked grain and the glycaemic response. Br Med J 297, 958-960.

17. Rebello CJ, Chu Y, Johnson WD, et al. (2014) The role of meal viscosity and oat beta-glucan characteristics in human appetite control: a randomized crossover trial. Nutr J 13, 49.

18. Wolever TMS \& Jenkins DJA (1986) The use of the glycemic index in predicting the blood glucose response to mixed meals. Am J Clin Nutr 43, 167-172.

19. Wolever TMS (2004) Effect of blood sampling schedule and method calculating the area under the curve on validity and precision of glycaemic index values. Br J Nutr 91, 295-300.
20. University of Sydney (2017) GI foods advanced search. http:// www.glycemicindex.com (accessed August 2018).

21. Wolever TMS, van Klinken BJ-W, Spruill SE, et al. (2016) Effect of serving size and addition of sugar on the glycemic response elicited by oatmeal: a randomized, cross-over study. Clin Nutr ESPEN 16, 48-54.

22. Christensen NJ, Ørskov H \& Hansen AP (1972) Significance of glucose load in oral glucose tolerance tests. Acta Med Scand 192, 337-342.

23. Wolever TMS \& Bolognesi C (1996) Source and amount of carbohydrate affect postprandial glucose and insulin in normal subjects. J Nutr 126, 2798-2806

24. Lee BM \& Wolever TMS (1998) Effect of glucose, sucrose and fructose on plasma glucose and insulin responses in normal humans: comparison with white bread. Eur J Clin Nutr $\mathbf{5 2}$, 924-928.

25. Wolever TMS, Gibbs AL, Spolar M, et al. (2006) Equivalent glycemic load (EGL): a method for quantifying the glycemic responses elicited by low carbohydrate foods. Nutr Metab (Lond) 3, 33.

26. Wolever TMS (2006) The Glycaemic Index: A Physiological Classification of Dietary Carbohydrate. Wallingford, UK: CABI.

27. Wolever TMS, Vorster HH, Björk I, et al. (2003) Determination of the glycaemic index of foods: interlaboratory study. Eur J Clin Nutr 57, 475-482.

28. Andersson AAM \& Börjesdotter D (2011) Effects of environment and variety on content and molecular weight of $\beta$-glucan in oats. J Cereal Sci 54, 122-128.

29. Herrera MP, Gao J, Vasanthan T, et al. (2014) $\beta$-Glucan content, viscosity, and solubility of Canadian grown oat as influenced by cultivar and growing location. Can J Plant Sci $\mathbf{9 6}$, 183-196.

30. Després J-P, Lemieux I \& Prud'homme D (2001) Treatment of obesity: need to focus on high risk abdominally obese patients. Br Med J 322, 716-720.

31. European Food Safety Authority (2012) Guidance on the scientific requirements for health claims related to appetite ratings, weight management, and blood glucose concentrations. EFSA J 10, 2604.

32. Health Canada (2013) Draft guidance document on food health claims related to the reduction in post-prandial glycaemic response. http://www.hc-sc.gc.ca/fn-an/consult/glycpostprandial/index-eng.php (accessed June 2013).

33. Wolever TMS (2017) Yogurt is a low-glycemic index food. J Nutr 147, 1462S-1467S.

34. Rebello CJ, Johnson WD, Martin CK, et al. (2016) Instant oatmeal increases satiety and reduces energy intake compared to a ready-to-eat oat-based breakfast cereal: a randomized crossover trial. J Am Coll Nutr 35, 41-49.

35. Anderson GH, Catherine NLA, Woodend DM, et al. (2002) Inverse association between the effect of carbohydrates on blood glucose and subsequent short-term food intake in young men. Am J Clin Nutr 76, 1023-1030. 